\title{
Modelo Matemático para Estimación de Eficiencias Fotónicas No-Intrínsecas en Reacciones Fotocatalíticas Heterogéneas
}

\author{
Miguel A. Mueses ${ }^{(1)(2)}$ y Fiderman Machuca-Martínez ${ }^{(2)}$ \\ (1) Facultad de Ingeniería, Programa de Ingeniería Química, Universidad de Cartagena, \\ Av. Del Consulado, 48-152, Edf. Ingeniería, Cartagena-Colombia. \\ (2) Escuela de Ingeniería Química, Grupo de Investigación GAOX, Universidad del Valle, \\ A. A. 25360, Cali-Colombia. (e-mail: mmueses@unicartagena.edu.co)
}

Recibido Jul. 29, 2011; Aceptado Sep. 30, 2011; Versión final recibida Nov. 20, 2011

\begin{abstract}
RESUMEN
Se formuló un modelo semiempírico para la estimación de eficiencias fotónicas en sistemas de reacción fotocatalítica heterogénea, en función de un factor pre-exponencial llamado eficiencia fotónica-intrínseca y un factor exponencial que cuantificó el efecto de carga de catalizador, geometría y concentración inicial de substrato. Los postulados y el modelo fueron validados en la fotodegradación de ácido dicloroacético en tres reactores fotocatalíticos heterogéneos a escala laboratorio, utilizando TiO2-P25, y radiación monocromática de baja energía. Se encontró que el modelo es altamente predictivo para eficiencias fotónicas con errores relativos menores al 1.8\%.
\end{abstract}

\section{Mathematical Model for Non-Intrinsic Photonic Yields in Heterogeneous Photocatalytic Reactions}

\begin{abstract}
A semi-empirical model was formulated to estimate the photonic efficiency of photocatalytic reactions in heterogeneous systems. This is a function of a pre-exponential factor called intrinsic photonic-efficiency and an exponential factor that quantified the effect of catalyst loading, geometry, and initial concentration of substrate. The assumptions and the model were validated on the photodegradation of dichloroacetic acid in three lab-scale heterogeneous photocatalytic reactors, using TiO2-P25 and low energy monochromatic radiation. It was found that the model is highly predictive for photonic efficiencies with relative errors below 1.8\%.
\end{abstract}

Keywords: photocatalysis, photonic efficiency, dichloroacetic acid, kinetic model 


\section{INTRODUCCIÓN}

El comportamiento cinético de los procesos de fotodegradación heterogénea utilizando semiconductores sólidos suspendidos es una función no solamente de la constante de velocidad de reacción intrínseca, de la energía de activación y de la naturaleza química del sistema; sino también de dos parámetros fotocinéticos denominados, eficiencia fotónica y la velocidad volumétrica de absorción de fotones (Mueses et al., 2008; Zalazar et al., 2005; Cassano y Alfano, 2000).

La definición estándar de eficiencia fotónica para sistemas fotoquímicos, está ligada a un estado de referencia dado por estimaciones actinométricas en fase homogénea (Zalazar et al., 2005; Serpone, 1997), o por estimación de eficiencias aparentes que permiten extrapolar resultados en fase homogénea a sistemas heterogéneos (Brandi et al., 2003). Típicamente las eficiencias fotónicas son calculadas por ajuste de parámetros de velocidad de reacción global, dependientes de las configuraciones geométricas, las condiciones de reacción y del flujo luminoso (es decir no intrínsecos) (Baoshun Liu et al., 2010; Li Puma et al., 2010; Ibrahim Gaya et al., 2008), pero independientes de los procesos fotocatalíticos de generación de especies fotogeneradas, electrónes-huecos e-/h+, en la interface semiconductor/solución (Machuca et al., 2008; Zalazar et al., 2005; Cassano y Alfano, 2000). Las velocidades volumétricas de absorción de fotones pueden estimarse por modelos empíricos o semiempíricos (Colina et al., 2010) o por solución rigurosa de la ecuación de transporte para la transferencia radiativa, RTE, a utilizando el método de la ordenada discreta (Cassano y Alfano, 2000; Duderstadt y Martin, 1979).

Investigaciones recientes han mostrado que las condiciones de proceso, el tipo de reactor y la escala de operación, para un sistema específico de reacción y catalizador utilizado, afectan los rendimientos de degradación global. Para reactores fotocatalíticos con mayores superficies de absorción de radiación y aprovechamiento de la energía directa o difusa como CPCs, placa plana, reactores multi-tubulares presentan mayores rendimientos globales de fotodegradación, independiente del tipo de la concentración inicial del sustrato (Sagawe et al., 2010; Mueses et al., 2008; Dijkstra et al., 2003). En trabajos previos (Mueses et al., 2010; Mueses et al., 2008) se evaluó el efecto del cambio de geometría y la concentración inicial de substrato, sobre los parámetros cinéticos de fotodegradación de ácido dicloroacético, especialmente en las eficiencias fotónicas del proceso. Se encontró que para geometrías con colectores de radiación difusa y mayores áreas de absorción, la eficiencia fotónica y por tanto la velocidad de reacción, mejoraron notablemente; para una misma concentración inicial de substrato. El efecto de la concentración inicial para geometrías definidas, mostró que la eficiencia fotónica es baja para bajas concentraciones debido a los efectos de recombinación de los pares electrón/hueco fotogeneradas.

El modelado matemático de reactores y cinética de fotocatálisis heterogénea se enfoca principalmente en ecuaciones de balance de materia y energía radiante, ecuaciones empíricas de velocidad de reacción, descripción de campos radiantes y procesos de degradación de substratos orgánicos contaminantes (Cassano y Alfano 2000, Colina et al., 2009). No obstante, a pesar de que las evidencias experimentales han mostrardo la naturaleza no intrínseca de las eficiencias fotónicas (Baoshun Liu et al., 2010; Li Puma et al., 2010; Trujillo et al., 2010; Urh Cernigoj et al., 2009, Ibrahim Gaya et al., 2008), no se han encaminado en modelado de este parámetro.

En este trabajo se presenta un modelo semiempírico para la estimación de eficiencias fotónicas en sistemas de reacción fotocatalítica heterogénea, en función de un factor pre-exponencial llamado eficiencia fotónica-intrínseca y un factor exponencial que cuantificó el efecto de carga de catalizador, geometría y concentración inicial de substrato.

\section{METODOLOGÍA: FORMULACIÓN DEL MODELO DE EFICIENCIA FOTÓNICA nO-INTRÍNSECA}

Primer postulado: La eficiencia fotónica en función de la velocidad de fotodegradación se comporta exponencialmente. El comportamiento experimental de la eficiencia fotónica global de los procesos de fotodegradación, en función de la velocidad de reacción, presenta una tendencia 
exponencial creciente por influencia de la transferencia de carga interfacial de los $h+$ libres hacia la solución, para flujo de potencia fotoquímica constante (Monllor-Satoca et al., 2007; Mora-Sero et al., 2005; Lana et al., 2004).

Segundo postulado: Se supone que si la eficiencia fotonica tiene una tendencia exponencial, es posible plantear una estructura matemática con funciones de contribución análogas a las funciones de contribución de la ecuación de Arrehnius de cinética de reacciones no isotérmicas, pero con signo contrario (puesto que la ecuación de Arrehnius es exponencial decreciente y la eficiencia fotónica una exponencial creciente).

La aplicación de los dos postulados permite obtener un modelo matemático para la descripción de eficiencias fotónicas no-intrínsecas cuya estructura matemática es:

$\Phi_{g, i}=\phi^{E f f}\left(\delta_{i}^{g} \varphi_{i}^{E f f}\right)^{\eta} \operatorname{Exp}\left[A_{i}^{*} \frac{C_{C a t}}{\delta_{i}^{g} \varphi_{i}^{E f f}}\left(\frac{1}{C_{i, 0}}\right)\right]$

Siendo el factor exponencial una función de corrección de absorción de energía; con $\psi(\varphi, E)$ la eficiencia fotónica del proceso e igual a $\left(\varphi_{g, i}\right) ; \varphi^{E f f}$ el factor pre-exponencial, o eficiencia fotónica intrínseca que cuantifica las interacciones en la interface semiconductor/solución, la cual depende solamente de la naturaleza de la reacción (intrínseco). $A_{i}^{*}$ un parámetro de concentración inicial de la solución en (moles/gramo de catalizador), $C_{\text {Cat }}$ la concentración de catalizador (gramos/L), $\varphi^{E F f}{ }_{i}$ es la velocidad volumétrica global adimensional de absorción de fotones (Machuca et al., 2008) y $C_{i, 0}$ es la concentración inicial de substrato. Para esta función, el potencial variacional es la velocidad volumétrica global de absorción de fotones. $\eta$ una constante de ajuste para la función de energía. El parámetro $\delta^{g}{ }_{i}$ corresponde a un parámetro asociado a la geometría del reactor, definido como:

$\delta_{i}^{g}=\frac{\left(S_{H}^{I}\right)^{2}}{S_{D}^{I} S_{T}}$

Con $S_{H}^{\prime}$ la superficie de absorción de radiación directa, $S_{D}^{\prime}$ la superficie irradiada con radiación difusa y $S_{T}$ es la superficie total del absorbedor. Para reactores de placa plana o reactores concéntricos $\delta_{i}^{g}=1$. Para reactores tubulares sin colectores de radiación $\delta^{g}{ }_{i}=\left(S_{H}^{\prime} / S_{T}\right)^{2}$. Para un reactor CPC (reactor tubular con colectores parabólicos compuestos):

$$
\begin{aligned}
& S_{H}^{I}=\pi D L \\
& S_{T}=\frac{1}{2} \pi D^{2}\left(1+f_{g}\right)
\end{aligned}
$$

La superficie $S_{D}^{\prime}$ depende del ángulo de la involuta del colector y se obtiene experimentalmente. Con $D$ y $L$ el diámetro y la longitud del absorbedor respectivamente y $f_{g}$, la relación diámetro: longitud.

\section{RESULTADOS Y ANÁLISIS}

El modelo formulado puede ser expresado como una función lineal logarítmica en términos de la concentración inicial del componente:

$$
\ln \left(\Phi_{g, i}\right)=\ln \left(\phi_{i}^{*}\right)+A_{i}^{*} \frac{C_{C a t}}{\delta_{i}^{g} \varphi_{i}^{E f f}}\left(\frac{1}{C_{D C A, i}^{i n}}\right)
$$


Donde el punto de corte, corresponde a $\ln \left(\varphi^{*}{ }_{i}\right)$ y la pendiente a una función geométrica, para concentraciones de catalizador constante. Se define entonces $\varphi^{*}{ }_{i}$ como la eficiencia fotónica aparente para cada concentración:

$\phi_{i}^{*}=\phi^{E f f}\left(\delta_{i}^{g} \varphi_{i}^{E f f}\right)^{\eta}$

En la Figura 1, se muestra el comportamiento de los logaritmos neperianos de estas eficiencias fotónicas, evaluadas para un sistema de degradación fotocatalítica heterogénea de ácido dicloroacético para flujo luminoso constante, monocromático, con una carga de catalizador $\mathrm{TiO}_{2-}$ P25 (Evonik-Degussa) de 1g/L; aplicado en tres reactores experimentales: un CPC, un placa plana y un tubular (Mueses et al., 2008). Los coeficientes de correlación son superiores a 0.92 lo cual garantizó un buen comportamiento predictivo. A partir de estas curvas se obtuvieron los valores para $\varphi^{*}{ }_{i}$ y $A^{*}$, los cuales se enlistan en la Tabla 1.

Tabla 1. Valores ajustados para $\varphi_{i}^{*}$ y $A_{i}^{*}$.

\begin{tabular}{cccc}
\hline Reactor & $\phi_{i}^{*} \times 10^{3}$ & $A_{i}^{*}$ & $\mathrm{R}^{2}$ \\
\hline CPC & 5.082256 & -0.0675 & 0.993 \\
Tubular & 2.979499 & -0.0235 & 0.998 \\
Axial & 0.503967 & -0.0015 & 0.917 \\
\hline
\end{tabular}

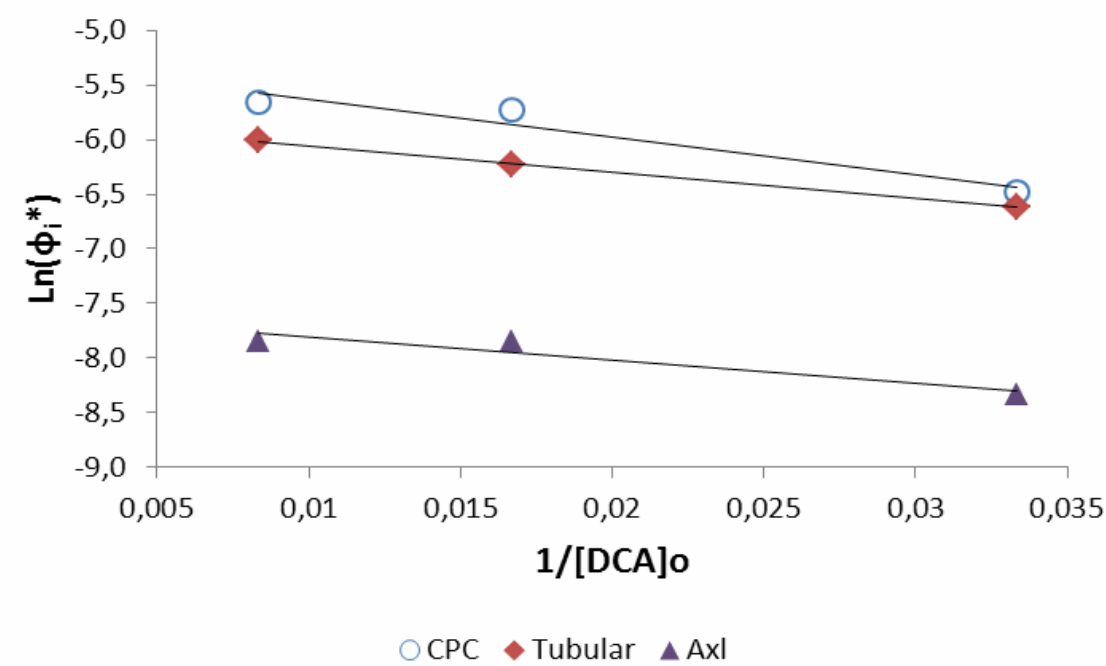

Fig. 1. Logaritmo natural de eficiencias fotónicas aparentes para la fotodegradación heterogénea de ácido dicloroacético.

El comportamiento lineal encontrado comprueba la validez de la estructura matemática del modelo formulado. Las eficiencias fotónicas (en logaritmo) obtenidas a partir de datos experimentales muestran una tendencia lineal con respecto al inverso de la concentración inicial del substrato, por tanto validan el primer postulado de la formulación. Evidencias experimentales de este comportamiento (exponencial) han sido reportadas en la literatura (Baoshun Liu et al., 2010; Li Puma et al., 2010; Ibrahim Gaya et al., 2008; Monllor-Satoca et al., 2007).

Ahora al evaluar el efecto de la geometría del sistema, a partir de las eficiencias fotónicas aparentes, los parámetros geométricos $\delta_{i}^{g}$ y la fracción de energía neta absorbida $\varphi^{\mathrm{Eff}}{ }_{i}$ es posible obtener la eficiencia fotónica intrínseca $\varphi^{E f f}$. Para eso se consideró una dependencia logarítmica entre las eficiencias fotónicas aparentes e intrínseca, en función del producto $\left(\delta^{g}{ }_{i} \varphi^{\mathrm{Eff}}{ }_{i}\right)$, así:

$\log \left(\phi_{i}^{*}\right)=\log \left(\phi^{E f f}\right)+\eta \log \left(\delta_{i}^{g} \varphi_{i}^{E f f}\right)$ 
En la Figura 2 se muestra el comportamiento y en la Tabla 2 los valores obtenidos en el ajuste.

Tabla 2. Valores ajustados para $\delta^{g}{ }_{i}$ y $\left(\delta^{g}{ }_{i} \varphi^{E f f}\right)$.

\begin{tabular}{ccc}
\hline Reactor & $\delta_{i}^{g}$ & $\delta_{i}^{g} \varphi_{i}^{\text {Eff }} \times 10^{3}$ \\
\hline CPC & 0.882353 & 1.958605 \\
Tubular & 0.441177 & 0.979301 \\
Axial & 0.249998 & 0.069793 \\
\hline
\end{tabular}

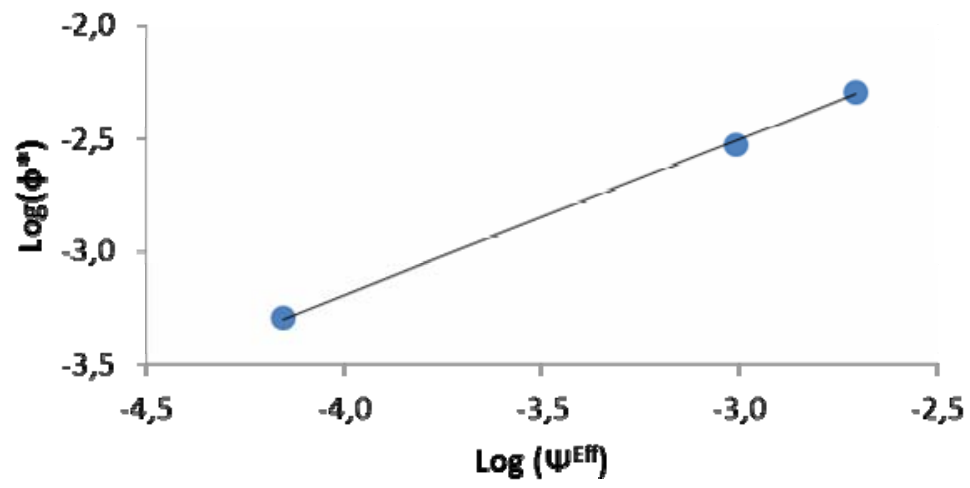

Fig. 2. Logaritmo natural de eficiencias fotónicas aparentes en función de los parámetros de absorción de energía radiante

El ajuste de la función anterior, arrojó un coeficiente de correlación de 0.999. Los valores obtenidos para la eficiencia fotónica intrínseca del ácido dicloroacético independiente de la geometría fue de $\varphi^{E f f}=0.36029321$ (mol/Einstein) y $\eta=0.68744477$ (adimensional). El valor numérico de la eficiencia fotónica encontrada es consistente en el rango de valores típicos para eficiencias globales reportadas en la literatura para DCA (Sagawe et al., 2010; Machuca et al., 2008; Zalazar et al., 2005) no obstante es un valor inédito para la literatura fotocatalítica. Su valor específico equivalente a la velocidad de generación de duplas e-/h+ en la etapa de activación del semiconductor, además es consistente físicamente debido a que relaciona las moles de ácido dicloroacético transformadas por unidad de energía absorbida por las partículas de catalizador.

El coeficiente de correlación del modelo comparado con datos de eficiencias fotónicas para esta sustancia evaluadas por métodos convencionales de ajuste de parámetros foto-cinéticos (Machuca et al., 2008) fue de 0.997. La Figura 3 presenta un grafico de dispersión con estas predicciones. Los errores relativos en la predicción fueron menores al 1.8\% lo cual garantizó la capacidad predictiva del modelo.

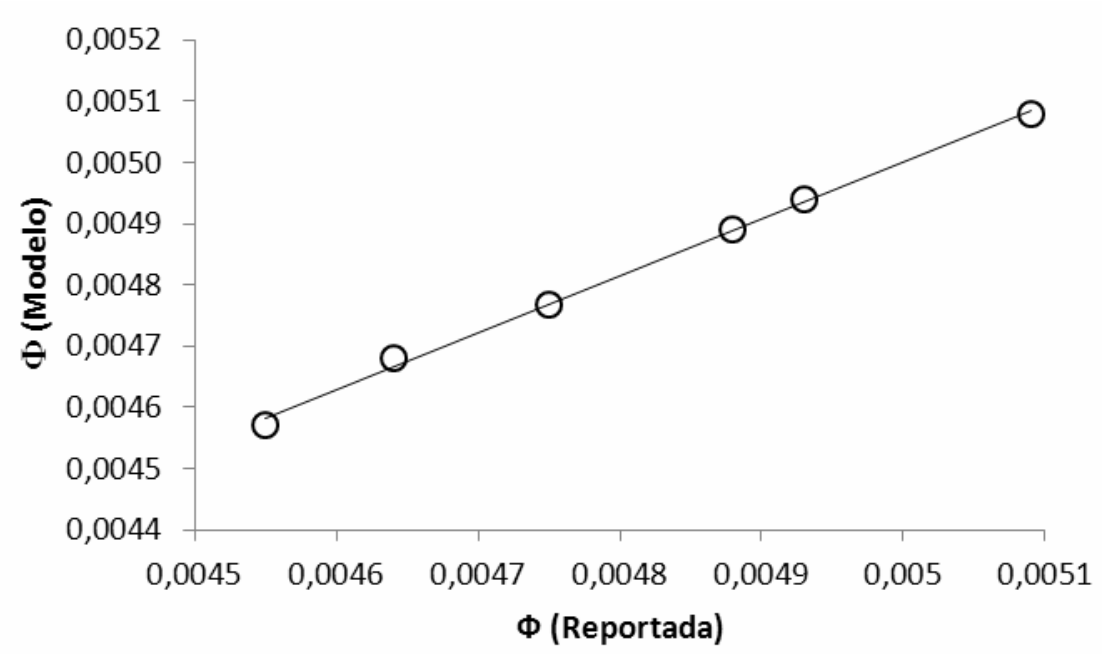

Fig. 3. Capacidad predictiva del modelo para eficiencias fotónicas reportadas por Machuca et al., 2008 
En la Figura 4 se muestra el algoritmo utilizado para la estimación de los parámetros fotónicos, el cual es un esquema de la metodología propuesta por Machuca y colaboradores en 2008:

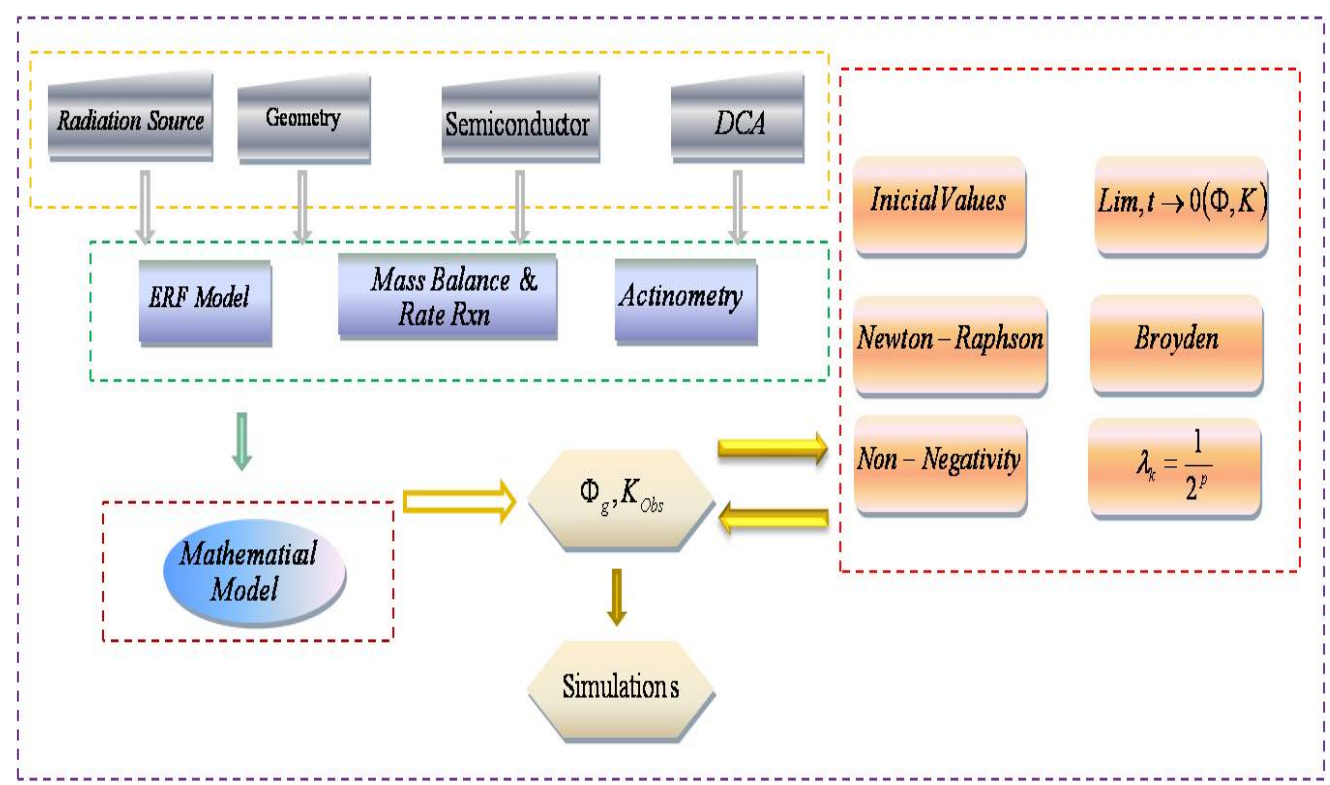

Fig. 4. Algoritmo de estimación de eficiencias fotónicas no intrínsecas.

Finalmente se encontró que el parámetro geométrico $A_{i}^{*}$ se correlaciona con el parámetro $\delta^{g}{ }_{i}$ de los reactores evaluados, mediante la siguiente función:

$$
A_{i}^{*}=0.024208\left(\delta_{i}^{g}\right)^{2}-0.131795\left(\delta_{i}^{g}\right)+0.029969
$$

Numéricamente las eficiencias fotónicas no-intrínsecas fueron calculadas utilizando un método modificado de mínimos cuadrados no lineales, acoplado al algoritmo de Newton-Raphson con un parámetro tipo Broyden (Mueses y Machuca, 2010). Se consideró una restricción matemática denominada eficiencia fotónica no negativa, que ayudó a la velocidad de convergencia del sistema. En la Figura 5, se muestra la predicción a diferentes condiciones de operación y geometrías de reacción, reflejadas en la velocidad volumétrica global de absorción de fotones (OVRPA) (Machuca et al, 2008), de eficiencias fotónicas para fotodegradación heterogénea de ácido dicloroacético, utilizando datos experimentales obtenidos por Mueses y colaboradores (Mueses et al, 2008).

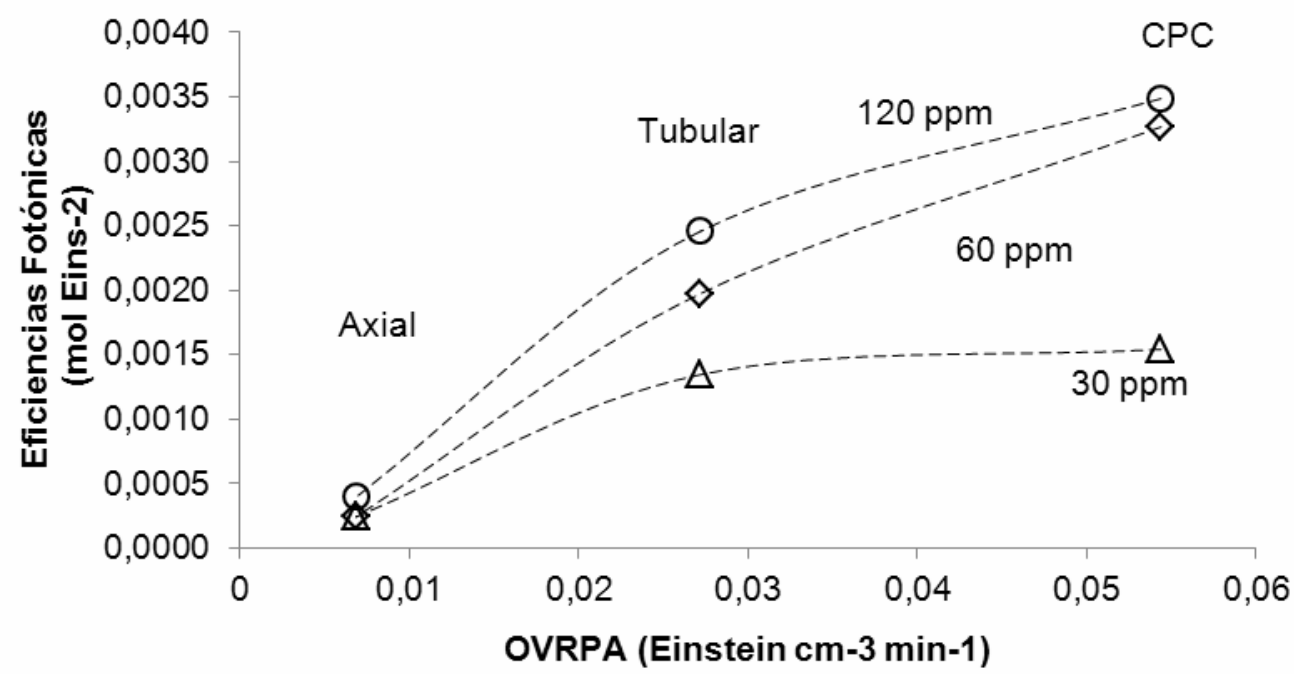

Fig. 5. Eficiencias fotónicas en función del la velocidad global de absorción de fotones para tres geometrías de reacción y condición inicial de substrato. 
Es evidente que el modelo formulado permite estimar el comportamiento de la eficiencia fotónica y describir físicamente la naturaleza del proceso de degradación. Para el TiO2-P25, la actividad e inducción de las duplas electrón-hueco, será afectada por la OVRPA del sistema. A OVRPA altas, la excitación superficial será alta y por tanto, la generación de duplas y radicales hidroxilo, evidenciando altas eficiencias fotónicas. En contraposición, OVRPA bajas inducen menos reacciones redóx, menor transformación de moléculas, mayor probabilidad de recombinación (e$/ \mathrm{h}+$ ) y por tanto, menores eficiencias fotónicas.

El modelo formulado puede utilizarse en modelos matemáticos de ecuaciones cinéticas de velocidad de reacción de distintas geometrías para la predicción y simulación de procesos de fotodegradación, debido a su carácter intrínseco.

\section{CONCLUSIONES}

Se formuló un modelo aproximado para la estimación de eficiencias fotónicas no-intrínsecas en sistemas de reacción fotocatalítica heterogénea, basado en un parámetro de eficiencia fotónica intrínseca independiente del tipo de reactor; y el producto de dos funciones, una de energía de absorción de fotones y otra de corrección de absorción por efecto de la carga de catalizador, la geometría del sistema y la concentración inicial de substrato. Los postulados fueron validados utilizando ácido dicloroacético como sustancia patrón. Se encontró que el modelo es altamente predictivo para eficiencias fotónicas de procesos de fotodegradación de este componente con errores relativos menores al $1.8 \%$.

\section{AGRADECIMIENTOS}

Los autores agradecen a la Universidad del Valle (Colombia) con el proyecto 2520 "Modelamiento y Simulación de la Cinética Intrínseca de Reacciones Fotocatalíticas Heterogéneas" por la financiación del proyecto y al Programa de Doctorados Nacionales de COLCIENCIAS (Colombia), por la financiación de estudios doctorales. Mueses agradece adicionalmente a la Universidad de Cartagena (Colombia) por la financiación en la publicación de este trabajo.

\section{REFERENCIAS}

Baoshun, L., Z. Xiujian, A Kinetic Model for Evaluating the Dependence of the Quantum Yield of Nano- $\mathrm{TiO}_{2}$ Based Photocatalysis, Electrochemica Acta: 55, $4062-4070$ (2010).

Brandi, R. J., M. Citroni, O. Alfano y A. Cassano, Absolute Quantum Yields in Photocatalytic Slurry Reactor, Chemical Engineering Science: 58, 979-985 (2003).

Cassano, A. y O. Alfano, Reaction Engineering of Suspended Solid Heterogeneous Photocatalytic Reactors, Catalysis Today: 58, 167-197 (2000).

Cernigoj, U., U. Lavrencic, P. Trebse y M. Sarakha, Determination of Catalytic Properties of $\mathrm{TiO}_{2}$ Coatings Using Aqueous Solutions, Journal of Photochemistry and Photobiology A: Chemistry: 201 (2-3), 142-150 (2009).

Colina J., F. Machuca, y G. Li Puma, Photocatalytic Mineralization of Commercial Herbicides in a Pilot-Scale Solar CPC Reactor: Photoreactor Modeling and Reaction Kinetics Constants Independent of Radiation Field, Environmental Science of Technology: 43, 8953-8960 (2009).

Colina J., F. Machuca y G. Li Puma, Radiation Absorption and Optimization of Solar Photocatalytic Reactors for Environmental Applications, Environmental Science of Technology: 44, 5112-5120 (2010).

Dijkstra, M., E. Koerts, A. Beenackers y J. Wesselingh, Performance of Immobilized Photocatalytic Reactors in Continuous Mode, AIChE Journal: 49 (3), 734-744 (2003). 
Duderstadt, J. y R. Martin, Transport Theory, Wiley, 421-422, New York, USA (1979).

Gaya, I. y H. Abdul, Heterogeneous Photocatalytic Degradation of Organic Contaminants Over $\mathrm{TiO}_{2}$, Journal of Photochemistry and Photobiology C: Photochemistry Reviews: 9, 1-12 (2008).

Lana, T., R. Gómez, M. González y P. Salvador, A Kinetic Model for Distinguishing between Direct and Indirect Hole Transfer in the Heterogeneous Photooxidation of Dissolved Organics on $\mathrm{TiO}_{2}$ Nanoparticle Suspensions, Journal of Physical Chemistry B: 108, 20278-20290 (2004).

Li Puma, G., V. Puddu, K. Tsang y A. Gora, Photocatalytic Oxidation of Multicomponent Mixtures of Estrogens (Estrone (E1), 17-Estradiol (E2), 17-Ethynylestradiol (EE2) and Estriol (E3) Under UVA and UVC Radiation, Applied Catalysis B: Environmental: 99, 388-397 (2010).

Monllor-Satoca, D., R. Gómez, M. González y P. Salvador, The Direct-Indirect Model: An Alternative Kinetic Approach in Heterogeneous Photocatalysis Based on the Degree of Interaction of Dissolved Pollutant Species with the Semiconductor Surface, Catalysis Today: 129, 247-255 (2007).

Mora-Sero, I., T. Lana, J. Bisquert, A. Pitarch, R. Gómez y P. Salvador, Photoelectrochemical Behavior of Nanostructured $\mathrm{TiO}_{2}$ Thin-Film Electrodes in Contact with Aqueous Electrolytes Containing Dissolved Pollutants, Journal of Physical Chemistry B: 109, 3371-3380 (2005).

Machuca, F., J. Colina y M. Mueses, Determination of Quantum Yield in a Heterogeneous Photocatalytic System Using a Fitting-Parameters Model", Journal of Advanced Oxidation Technologies: 11 (1), 42-48 (2008).

Mueses, M., A. Arce, J. Colina y F. Machuca, Effect of Reactor Size on the $\mathrm{TiO}_{2}$-Based Photodegradation of Dichloroacetic Acid with Solar CPCs", in "The $15^{\text {th }}$ International Conference on TiO2 Photocatalysis: Fundamentals and Applications", San Diego, CA, USA (2010).

Mueses, M., J. Colina, F. Machuca-Martínez, Degradación Fotocatalítica de Ácido Dicloroacético al Aplicar un Campo de Radiación de Baja Energía, Ingeniería \& Desarrollo: 24, 34-47 (2008).

Mueses M. y F. Machuca, Una Solución de Rachford-Rice para Sistemas Multifases Aplicando el Método de Newton-Raphson, Un Parámetro de Broyden y el Flash Negativo, Información Tecnológica: 21 (4), 3-10 (2010).

Sagawe, G., M. Satuf, R. Brandi, J. Muschner, C. Federer, O. Alfano, D. Bahnemann y A. Cassano, Analysis of Photocatalytic Reactors Employing the Photonic Efficiency and the Removal Efficiency Parameters: Degradation of Radiation Absorbing and Nonabsorbing Pollutants, Industrial \& Engineering Chemistry Research: 49 (15), 6898-6908 (2010).

Serpone, N., "Relative Photonic Efficiencies and Quantum Yields in Heterogeneous Photocatalysis, Journal of Photochemistry and Photobiology A: Chemistry: 104, 1-12 (1997).

Trujillo, T., T. Safinski y A. Adesina, Oxidative Photomineralization of Dichloroacetic Acid in an Externally-Irradiated Rectangular Bubble Tank Reactor: Computational Fluid Dynamics Modeling and Experimental Verification Studies, Industrial \& Engineering Chemistry Research: 49 (15), 6722-6734 (2010).

Zalazar, C., R. Romero, C. Martin y A. Cassano, Photocatalytic Intrinsic Reaction Kinetics I: Mineralization of Dichloroacetic Acid, Chemical Engineering Science, 6052405254 (2005). 